\title{
Clinical Characteristics of Patients with Trigeminal Neuralgia Referred to Neurosurgery
}

\author{
Silvia RDT Siqueira \\ Manoel J Teixeirab \\ José TT Siqueirac
}

\section{ABSTRACT}

Objectives: To investigate the clinical characteristics of patients with trigeminal neuralgia referred to surgery in a center of reference.

Methods: We evaluated the general characteristics of 395 patients with trigeminal neuralgia referred to neurosurgery as treatment. They corresponded to 2 samples of 1984 and 2004. The EDOFHC protocol (Orofacial Pain Questionnaire) and the medical profile were used.

Results: In the first study (1984), with 290 patients, the higher prevalence was: women (57.3\%), white (95.5\%), with mean age of 62.5 . The most affected trigeminal branches were the maxillary and / or mandibular branches (65.5\%), and the right side was the most affected (57.6\%). From the second study (2004), with 105 patients, $57.1 \%$ were women, $75.2 \%$ white, with a mean age of 60.8 . The maxillary and / or mandibular branches (79.0\%) and the right side (69.5\%) were the most affected. Both samples had neurological abnormalities and systemic diseases (mainly cardiovascular).

Conclusions: General characteristics of these patients were similar to other samples of trigeminal neuralgia. Neurological findings were also present in patients with no previous surgical treatment for TN. Hypertension and cardiac diseases were also frequent and make the monitoring of the patients during crises necessary. (Eur J Dent 2009;3:207-212)

Key words: Hypertension; Neurosurgery; Orofacial pain; TMD; Trigeminal neuralgia.

a DDS, PhD, Assistant Professor at Escola de Artes, Ciencias e Humanidades and member of the Orofacial Pain Team, Dentistry Division, Hospital das Clinicas, Medical School, University of São Paulo, SP, Brazil.

b MD, PhD, Head of the Pain Center of Hospital das Clinicas, Chairman of Neurosurgery, Neurology Department, Medical School, University of São Paulo, SP, Brazil.

DDS, PhD, Head of the Orofacial Pain Team, Dentistry Division, Hospital das Clínicas, Medical School, University of São Paulo, SP, Brazil.

- Corresponding author: Silvia RDT de Siqueira Rua Carlos Weber, 1319 apto P 164 Vila Leopoldina 05303-000 Sao Paulo - SP - Brazil

Phone: 551191842878

E-mail: silviadowganahotmail.com

\section{INTRODUCTION}

Trigeminal neuralgia (TN) is a paroxysmal shock-like pain restricted to the innervation area of one or more trigeminal branches, often set off by light stimuli in a trigger zone. ${ }^{1,2}$ It is idiopathic, and imaging exams (computed tomography and/ or magnetic resonance) are necessary to exclude intracranial expansive lesions, which are present in $5 \%$ of cases. $^{3}$ TN causes severe compromise of the oral hygiene, habitual tasks and quality of life, and the patients often get depressed and desperate. ${ }^{1,4}$

In studies in USA, ${ }^{5}$ Italy $^{6}$ and France, ${ }^{7} 155$ habitants in each 1.000.000 are affected. General 
characteristcs are similar among countries and include a mean of age around 60 years old, being more often in the female gender, right side, maxillary and / or mandibular trigeminal branches, in opposite to post-herpetic neuralgia, more common at the ophthalmic branch. TN is the first symptom of multiple sclerosis in $1-4 \%$, and it affects $5-10 \%$ of multiple sclerosis patients. ${ }^{1}$ In these cases, it is usually bilateral.

Patients are often referred to neurosurgery because of clinical treatment failure, and the duration of the disease usually influences that. On the other side, other diseases are common in the elderly and can cause complications during and after the surgery. ${ }^{2}$

Thus, the objective of this study was to investigate the clinical characteristics of patients with trigeminal neuralgia referred to neurosurgery in a center of reference.

\section{MATERIALS AND METHODS}

This study was based on the retrospective evaluation of 395 patients with trigeminal neuralgia referred to neurosurgical treatment at a general hospital, which is a center of reference for this kind of treatment for all parts of the country. The patients included in this study corresponded to all that were indicated for percutaneous procedures, including balloon compression of the trigeminal ganglion and radiofrequency rhizotomy. All of them underwent an evaluation of postoperative complications. ${ }^{8,9}$ All patients signed the informed consent and the protocol was approved by the Ethics Committee of the Hospital. They were referred to neurosurgery because of failure of the pharmacological treatment with anticonvulsants or because of collateral effects including allergy, hepatic disease and decrease in the number of leukocytes in blood count.

Patients were diagnosed according to the definition on the criteria of the International Association for the Study of Pain (IASP). ${ }^{10}$ These criteria were the edition for unification of pain diagnosis; for trigeminal neuralgia, it was based on the definition from 1776 of John Fothergill. The first 290 patients were evaluated between April, 1979 and June, 1984, and the last 105 patients were evaluated between June, 2003 and May, 2004.

Complementary investigation consisted of skull radiography and cranial tomography in all patients, which were normal in this sample.

Patients were evaluated considering their general characteristics, including gender, ages, color or race, and trigeminal neuralgia characteristics (side and branch affected). Associated diseases and other findings, including neurological abnormalities were also investigated, and data were crossed to verify possible correlations among the variables and between samples.

The instrument used was the EDOF-HC protocol (Orofacial Pain Clinic - Hospital das Clinicas), a standardized orofacial pain questionnaire to detail: 1) chief complaint, 2) general pain characteristics llocation, quality, duration, pain relief or pain triggering), 3) presence of headache and/or body pain, and 4) patient's medical history and co-morbidities. ${ }^{2}$

\section{Statistical analysis}

Data were analyzed in their frequencies, means and standard deviations, and the intervals of confidence and proportions. The chi-square test was used to compare data between the samples and the level of significance was of $5 \%$. The $T$ test was used to compare means between the samples.

\section{RESULTS}

First study - 290 patients 9

From 290 TN patients, 166 (57.3\%) were female and 124 (42.7\%) were male, which was statistically significant when compared to the other sample from $2004(P<.05) ; 277(95.5 \%)$ of patients were white, $7(2.4 \%)$ yellow and $6(2.1 \%)$ black. Three $(1.0 \%)$ patients had family history of $\mathrm{TN}$ and $3.4 \%$ patients had previous history of surgical treatment for TN, but because of pain recurrence were referred again to surgery.

Distribution of ages can be observed in Figure 1. Ages ranged from 17 to 88 years old, with a mean of 62.5 years old. There was a higher prevalence of the 7 th decade of life. There was no difference between the ages of female and male patients. Ages at the pain onset ranged from 12 to 85 years old. Mean duration of pain was 95.0 months. TN was more frequent at the right side $(167$ patients; $57.6 \%)$. It was bilateral in 17 (5.9\%) patients.

Figure 2 shows distribution of patients according to the trigeminal branch affected. The association of the maxillary and the mandibular 
branch $(\mathrm{V} 2+\mathrm{V} 3)$ was the most frequent $(28.0 \%)$, followed by the association of the ophthalmic and the maxillary branch $(\mathrm{V} 1+\mathrm{V} 2)(19.9 \%)$ and only V3 $(18.9 \%)$.

Systemic diseases were observed in 235 (81.0\%) patients (Table 1), and among them, heart diseases were the most common (71.8\%). The prevalence of hypertension was higher in the sample from 1984 when compared to the other one $(P<.05)$. The type of hypertension was not collected.

Trigger zones were observed in 212 (73.1\%) patients. Corneal reflex asymmetry was present in $6.8 \%$ of patients, and facial hypoesthesia in $29.6 \%$. From these cases, $4.7 \%$ of the patients with facial hypoesthesia and $12.8 \%$ of patients with corneal reflex asymmetry had no previous history of surgery at the face or for TN. On the other hand, $3.4 \%$ of patients had facial nerve deficit and $2.2 \%$ trigeminal motor abnormalities, and all of these patients had had previous surgical treatment for TN. Other neurological findings were Parkinson's disease in 3 patients, essential tremor in 3, previous vascular cerebral accident in 2, concomitant glossopharyngeal neuralgia TN in 2, vertebral-basilar malformation in 1 and previous parietal meningoma in 1 .

Facial spasm was observed in $8(2.8 \%)$ patients. It was ipsilateral in $4(50.0 \%)$ of them, and more frequent in patients with bilateral neuralgia $111.8 \%$ of cases) when compared to unilateral neuralgia (2.2\% of cases) $(P<.05)$.

Only three patients had initial failure with the balloon compression, and they were re-operated. They had no comorbidity associated and the trigeminal branches affected were $\mathrm{V} 3$ in 2 patients and V2-3 in one. All of them had the right side affected.

Second study - 200411

From 105 TN patients, 60 (57.1\%) were female

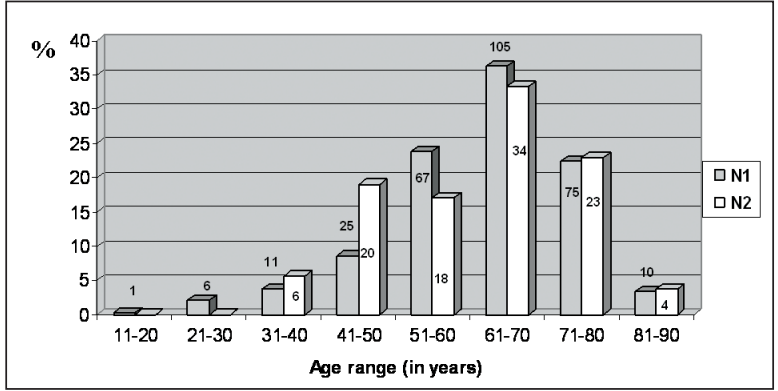

Figure 1. Distribution of patients according to the ages in percentages (N1=290, N2=105). and $45(42.9 \%)$ were male, which was statistically significant ( $P<.05) ; 79(75.2 \%)$ patients were white, $23(21.9 \%)$ black and $3(2.9 \%)$ yellow. Three $(2.9 \%)$ patients had family history of TN.

Ages ranged from 35 to 85 years old, with a mean of 60.8 years old (Figure 1). There was a higher prevalence of the $7^{\text {th }}$ decade of life. There was no difference between the mean of ages of female and male patients. Ages at the pain onset ranged from 24 to 78 years old. Mean duration of pain was 114.0 months.

$\mathrm{TN}$ was more often at the right side $(73$ patients $-69.5 \%)(P<.05)$. It was bilateral in $1(1.0 \%)$ patient.

Figure 2 shows the distribution of patients according to the trigeminal branch affected. The mandibular (V3) (29.5\%) and the maxillary (V2) $(29.5 \%)$ branches isolated were the most affected, followed by the association between both (20.5\%). These patients had more often an isolated trigeminal branch affected when compared to the first sample $(P<.001)$.

Systemic diseases were present in 75 (71.4\%) patients (Table 1). They had lower frequency of heart diseases $(41.8 \%)$ than the other group of patients $(P<.05)$.

Trigger zones were observed in 90 (85.7\%) patients (Table 2). Corneal reflex asymmetry was higher in this group and occurred in $20.1 \%$; facial hypoesthesia occurred in $27.3 \%$, which was similar to the other group; $9.9 \%$ of the patients with hypoesthesia and $13.6 \%$ of the patients with corneal reflex asymmetry had no previous history of surgery at the face or for TN. There were no other relevant neurological findings in this sample.

All patients with multiple sclerosis had unilateral TN. Despite these differences between both samples about branch affected and prevalence of systemic diseases, we could not find any other statistical differences. The general

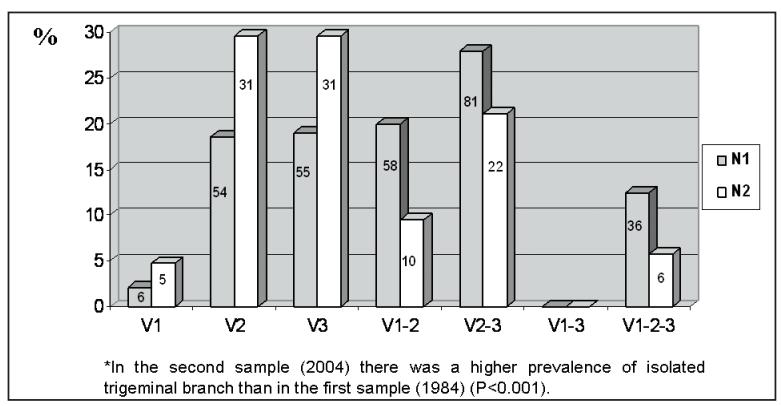

Figure 2. Distribution of patients according to the trigeminal branch affected in percentages (N1=290, N2=105). 
characteristics as gender and ages were similar, with the balloon compression of the trigeminal and the neurological abnormalities were not associated to any other finding, as side affected, trigeminal branch or co-morbidity.

ganglion. Only one patient had initial failure with the balloon compression, and was re-operated.

All patients in this sample were operated at V3, at the right side.

Table 1. Trigeminal neuralgia : relevant clinical findings $(N 1=290, N 2=105)$.

\begin{tabular}{lcc}
\hline Clinical findings & $\mathrm{N} 1$ & $\mathrm{~N} 2$ \\
& $\mathrm{~N}(\%)$ & $\mathrm{N}(\%)$ \\
\hline Breathing chronic insufficiency & $14(4.8)$ & - \\
\hline Cardiac arrhythmia & $11(3.8)$ & - \\
Myocardial ischemia & $17(5.9)$ & $4(3.8)$ \\
Cardiac insufficiency & $25(8.6)$ & $20(19.0)$ \\
Moderate arterial hypertension* & $107(36.9)$ & $20(19.0)$ \\
Severe arterial hypertension* & $40(13.8)$ & - \\
Duodenal ulcer & $5(1.7)$ & $11(10.5)$ \\
Diabetes mellitus & $14(4.8)$ & - \\
Nefro-insufficiency & $2(3.4)$ & $2(1.9)$ \\
Multiple sclerosis & - & $2(1.9)$ \\
Migraine & - & $3(2.8)$ \\
Depression & - & $1(1.0)$ \\
Reumathoid arthritis & - & $10(9.9)$ \\
Other & $235(81.0)$ & $75(71.4)$ \\
TOTAL & & \\
*Brazilian Academy of Hypertension & & \\
\hline
\end{tabular}

*Brazilian Academy of Hypertension

Table 2. Trigeminal neuralgia: trigger zones location (N2=105).

\begin{tabular}{lc}
\hline Trigger zones & $\mathbf{N}(\%)$ \\
\hline Lips & $24(22.9)$ \\
Nose wing & $19(18.1)$ \\
Alveolar bone & $18(17.1)$ \\
Zigoma & $9(8.6)$ \\
Head & $5(4.8)$ \\
Tooth & $4(3.8)$ \\
Lips and nose wing & $4(3.8)$ \\
Chin & $3(2.9)$ \\
Vestibular area & $1(1.0)$ \\
Cheek & $1(1.0)$ \\
Tongue & $1(1.0)$ \\
Eyelid & $1(1.0)$ \\
Nonspecific & $15(14.3)$ \\
TOTAL & $105(100.0)$ \\
\hline
\end{tabular}

European Journal of Dentistry 


\section{DISCUSSION}

$\mathrm{TN}$ is a rare excruciating disease with long-term treatment and frequent neurosurgical indication. This study determined the general characteristics of $395 \mathrm{TN}$ patients in a Brazilian population from two samples (1984 and 2004). Its prevalence, epidemiology and general characteristics were similar to other countries..$^{1,4-6} \mathrm{TN}$ is more frequent in female patients, which can be due to a longer life expectation of women, and $\mathrm{TN}$ is more common in the elderly. ${ }^{11}$ Rarely familiar cases are reported, ${ }^{12}$ and it happened in $1.0 \%$ of the patients at 1984 and in $2.9 \%$ at 2004 . There are no studies that determined ethnical differences before, and although in this sample there was a higher prevalence of white patients, this issue should be investigated with samples from different parts of the world.

The right side was more affected than the left, and V2 and / or V3 were the most affected branches, which corresponds to other studies and is opposite to post-herpetic neuralgia. ${ }^{13}$ It is interesting that the first group of patients had more trigeminal branches association than the second group $(P<.001)$. The reason for it is unknown, but the affection of adjacent branches as long as the disease progresses until the referral for surgery might be an explanation. ${ }^{11}$ Patients at the second sample were only referred to surgery after complete failure of pharmacological treatment, and thus the disease progressed affecting more branches. Due to neurosurgical complications, neurosurgery was usually delayed and only indicated when there was complete failure of pharmacological treatment, but with the new non invasive techniques after the $80 \mathrm{~s}$, it started to be performed earlier. ${ }^{12}$

Neurological findings labnormalities of the corneal reflex, facial spasm and facial hypoesthesial were present also in patients with no previous history of surgery, which corresponded to other studies that found discrete sensorial abnormalities in TN. ${ }^{14,15}$ Trigeminal central sensitization occurs in any chronic pain, and sensorial abnormalities can happen. In these samples, they were not associated to any other findings of general characteristics, co-morbidities, surgical modality or other post-operative complications, which probably means that they are random, but future studies should investigate it to confirm that. The neurological findings are not implicated in the referral for surgery and thus not biases in this study. ${ }^{7}$

Hypertension and cardiac diseases were frequent in these samples and higher in the first group of patients $(P<.05)$. Although this rate is slightly higher than the expected for the Brazilian general population at the same ages of our study, ${ }^{16}$ it is possible that the difference is due to imperfect medical records at that evaluation time. It is important to remind that intense pain can cause neurovegetative abnormalities and blood pressure increase can happen due to that when patients are in crises, and patients that already have hypertension can have severe complications due to that. On the other hand, the crises could occur more frequently in higher blood pressure episodes because of the vascular compression etiology of TN. The relevance this fact is controversial, and some authors have reported that it could be a coincidence in these patients. ${ }^{17}$ $\mathrm{TN}$ is very excruciating and is considered one of the worst pains, with subtle and intense crises, and cardiac compromise could happen after a long period of suffering. ${ }^{2}$ This is important because these patients should be monitored and supports the early indication of surgery when it is necessary to prevent these complications; there are many studies in the literature focused on that. ${ }^{18-22}$ The differences of prevalence of depression and migraine in Table 1 are small and can be due to the imperfect data collection in the first sample, at the medical records.

In this study, 2 different samples of well documented patients with TN were evaluated in order to investigate their clinical characteristics. They correspond to two distinct periods of data collection. The option of maintenance of their data separated was not as comparison but because of the interval of time between them. Therefore, when analyzing the data, there were differences in the prevalence of systemic diseases and trigeminal branch affected, and that was the reason we opted in tabling it. This might be a limitation of the study, but as we could observe both samples of patients with trigeminal neuralgia are homogeneous and similar in their characteristics. Other postoperative complications that did not differ between them can be observed in our previous publications..$^{9,11}$ 


\section{CONCLUSIONS}

The general characteristics were similar than other studies about gender, ages, branch and side affected. Neurological findings were present also in patients with no previous surgical treatment for TN. Hypertension and cardiac diseases were also frequent and make the monitoring of the patients during crises necessary.

\section{REFERENCES}

1. Rothman KJ, Monson RR. Epidemiology of trigeminal neuralgia. J Chron Dis 1973;26:2-12.

2. Siqueira SRDT, Nóbrega JCM, Valle LBS, Teixeira MJ, Siqueira JTT. Idiopathic trigeminal neuralgia: Clinical aspects and dental procedures. Oral Surg Oral Med Oral Pathol Oral Radiol Endod 2004;98:311-315.

3. Benedittis G, Bernasconi V, Ettore G. Tumours of the fifth cranial nerve. Acta Neurochir 1977;38:37-64.

4. Patterson CW. Trigeminal neuralgia - a dental diagnosis challenge. Northwest Dent 1999;78:19-24.

5. Beaver DL. Electron microscopy of the gasserian ganglion in trigeminal neuralgia. J Neurosurg 1963;20:138-150.

6. Buscaino GA. Fisiologia della nevralgia essenziale del trigemino. Acta Neurol 1980;35:137-144.

7. Zakrzewska JM. Trigeminal eye and ear pain. In: Wall PD, Melzack R, editors. Textbook on pain. 4th ed. Edinburgh London: Churchill Livingstone 1999:739-59.

8. Siqueira SRDT, Lara C, Nóbrega JCM, Siqueira JTT, Teixeira MJ. Sensitivity evaluation of patients with idiopathic trigeminal neuralgia that underwent functional neurosurgery as treatment. The Pain Clinic 2006;18:87-92.

9. Teixeira MJ, Siqueira SRDT, Almeida, GM. Percutaneous radiofrequency rhizotomy and neurovascular decompression of the trigeminal nerve for the treatment of facial pain. Arq Neuropsiquiatr 2006;64:983-989.

10. Merskey H, Bogduk N. Classification of chronic pain. $2^{a}$. Ed. Seattle: IASP Press, 1994.

11. Siqueira SR, Nóbrega JC, Teixeira MJ, Siqueira JT. Masticatory problems after balloon compression for trigeminal neuralgia: a longitudinal study. J Oral Rehabil 2007;34:88-96.

12. Barraquer-Bordas L. Sobre la herencia y etiopatogenia del tic doloroso trigeminal. Arq Neuropsiquiatr 1949;7:241-263.

13. Auld AW, Buermann A, Gables A. Trigeminal neuralgia in six members of one generation. Arch Neurol 1965;13:194198.

14. Brown GR. Herpes zoster: correlation of age, sex, distribution, neuralgia, and associated disorder. South Med $1976 ; 69: 576-578$
15. Synai VJ, Bonamico LH, Dubrovsky A. Subclinical abnormalities in trigeminal neuralgia. Cephalalgia 2003;23:541-544.

16. Zaslavsky C, Gus I. Idoso. Doença cardíaca e comorbidades. Arq Bras Cardiol 2002;79:635-639.

17. Teruel A, Ram S, Kumar SK, Hariri S, Clark GT. Prevalence of hypertension in patients with trigeminal neuralgia. $J$ Headache Pain. 2009. In press.

18. Schaller B. Trigemino-cardiac reflex during microvascular trigeminal decompression in cases of trigeminal neuralgia. J Neurosurg Anesthesiol 2005; 17:45-48.

19. Dominguez J, Lobato RD, Rivas JJ, Gargallo MC, Castells V, Gozalo A, Sarabia R. Changes in systemic blood pressure and cardiac rhythm induced by therapeutic compression of the trigeminal ganglion. Neurosurg 1994;34:422-428.

20. Brown JA, Preul MC. Trigeminal depressor response during percutaneous microcompression of the trigeminal ganglion for trigeminal neuralgia. Neurosurg 1988;23:745748.

21. Tatli M, Satici O, Kanpolat Y, Sindou M. Various surgical modalities for trigeminal neuralgia: literature study of respective long-term outcomes. Acta Neurochir 2008; 150:243-255.

22. Natarajan M. Percutaneous trigeminal ganglion balloon compression: experience in 40 patients. Neurol India 2000;48:330-332. 